BRX-TH-520

\title{
Shortcuts to high symmetry solutions in gravitational theories
}

\author{
S. Deser and Bayram Tekin ${ }^{12}$ \\ Department of Physics, Brandeis University, Waltham, MA 02454, USA
}

\begin{abstract}
We apply the Weyl method, as sanctioned by Palais' symmetric criticality theorems, to obtain those -highly symmetric -geometries amenable to explicit solution, in generic gravitational models and dimension. The technique consists of judiciously violating the rules of variational principles by inserting highly symmetric, and seemingly gauge fixed, metrics into the action, then varying it directly to arrive at a small number of transparent, indexless, field equations. Illustrations include spherically and axially symmetric solutions in a wide range of models beyond $D=4$ Einstein theory; already at $D=4$, novel results emerge such as exclusion of Schwarzschild solutions in cubic curvature models and restrictions on "independent" integration parameters in quadratic ones. Another application of Weyl's method is an easy derivation of Birkhoff's theorem in systems with only tensor modes. Other uses are also suggested.
\end{abstract}

\footnotetext{
${ }^{1}$ e-mail: deser,tekin@brandeis.edu

${ }^{2}$ Address after July 15, Physics Department, Middle East Technical University, 06531, Ankara, Turkey.
} 


\section{Introduction}

In his classic monograph on general relativity [1], Weyl derived the Schwarzschild metric by a very elegant method leading to just two scalar field equations instead of the usual tensorial set. The simplification was achieved by choosing the input metric not only to exhibit spherical symmetry and a priori time-independence but also to select a particular gauge, Schwarzschild coordinates; in our (slightly different) parametrization,

$$
d s^{2}=-a(r) b(r)^{2} d t^{2}+\frac{d r^{2}}{a(r)}+r^{2} d \Omega_{2}
$$

where $d \Omega_{2}$ is the 2 -sphere element, the Einstein action simply takes the form

$$
I=\int d^{4} x \sqrt{-g} R \rightarrow \int_{0}^{\infty} d r r(a-1) b^{\prime}
$$

The two Euler-Lagrange equations obtained by varying the dependent variables $(a, b)$ in $(2)$ are not only of first derivative order, but trivially integrable to the correct metric, $a=1-c_{1} / r$, $b=c_{2}$. It did not escape subsequent readers that this procedure seems to violate all the tenets

of variational principles: It inter-compares solely static ${ }^{3}$, spherically symmetric geometries and even specifies the gauge! The correctness of the results, however, suggests that that there must be something to this procedure. Indeed, a mere fifty years later, a mathematical justification ( and equally important, its limitations) was given by the symmetric criticality theorems of Palais [2]. We will not expound them here. ${ }^{4}$

Surprisingly, there has been very little application of this streamlined approach to GR, let alone to more complicated gravitational models involving higher curvature powers and generic dimension. Our aim here is the modest one of advertising the virtues of this technique through concrete generation of spherically and axially symmetric solutions in a variety of models, including matter and various string-generated corrections to Einstein theory such as the conformal Weyl action in $D=4$, higher powers of curvature, or Gauss-Bonnet terms in $D>4$. We mention

\footnotetext{
${ }^{3}$ This assumption is also tacit and unjustified in many textbooks.

${ }^{4}$ For a recent discussion in the framework of GR, see [3].
} 
several novel results: pure $R^{2}$ gravity in $D=4$ allows either Schwarzschild-deSitter (SdS) or Reissner-Nordstrom (RN) solutions but not both; the actions cubic in the Weyl tensor plus any lower power of curvature at $D=4$ do not permit the Schwarzschild metric; in the four dimensional $R_{\mu \nu \rho \sigma}^{2}$ model, the only solution of the form $-g_{00}=g^{r r}$ is $\mathrm{SdS}$.

We hope that further novel applications will be found. One domain is obviously that of 'brane' metrics. Another would exploit the fact that, under precisely defined circumstances, it is possible to generate correct solutions-along with metrics that are not correct-despite even more egregious violations of the ground rules; since it is easy to screen out non-solutions, one may hope to thereby obtain as yet unknown geometries in more complicated models. Separately, we will obtain the Birkhoff theorem, for models without scalar or vector modes, as another type of application . Also, depending how one chooses to parametrize the metric, one might generate solutions in other coordinates, although care is required here. More generally, we caution that the fine print must always be read with care.

\section{Spherical Symmetry}

The most general spherically symmetric metric in dimension $D$ and Schwarzschild gauge is

$$
d s^{2}=-a(r, t) b(r, t)^{2} d t^{2}+\frac{d r^{2}}{a(r, t)}+r^{2} d \Omega_{D-2}
$$

where $d \Omega_{D-2}$ is the $D-2$ sphere element and the two functions $(a, b)$ depend on $(r, t)$ a priori. This "gauge" is well-known to be very special in that it is not so much a gauge choice but rather a natural expression of the symmetry orbits [2]. We have already noted its advantage in GR of reducing the derivative order of the action $(2) . \quad 5$

Let us start with the most elementary example of cosmological GR,

$$
I=\int d^{D} x \sqrt{-g}(R+\Lambda)
$$

\footnotetext{
${ }^{5}$ An example of illegal gauge choice is to further reduce the $(r, t) 2$-space to a single function, say to conformally flat form with $t$-independence. We know that the Schwarzschild metric cannot be so expressed.
} 
The scalar curvature and volume form are respectively

$$
\begin{aligned}
R & =a^{\prime \prime}+\frac{2 a b^{\prime \prime}}{b}+\frac{3 a^{\prime} b^{\prime}}{b}+2(D-2) \frac{1}{r}\left(a^{\prime}+\frac{a b^{\prime}}{b}\right)+(D-2)(D-3) \frac{1}{r^{2}}(a-1) \\
& +\frac{1}{a^{2} b^{2}}\left(\ddot{a}-2 \frac{\dot{a}^{2}}{a}-\frac{\dot{a} \dot{b}}{b}\right), \quad \sqrt{-g}=b r^{D-2} .
\end{aligned}
$$

The time derivatives all drop out in the action (4)

$$
I=(D-2) \int_{0}^{\infty} d r r^{D-3}\left(a-1-\frac{\Lambda r^{2}}{D-2}\right) b^{\prime}
$$

absence of any time derivatives in the integrated form is Birkhoff's theorem. It is now also obvious that the solution of (6) is $\mathrm{SdS}$,

$$
a(r)=1+\frac{c_{1}}{r^{D-3}}+\frac{\Lambda}{D-2} r^{2}, \quad b(r)=c_{2}
$$

for $D>3$, and the conical SdS

$$
a=c_{1}+\Lambda r^{2}, \quad b=c_{2},
$$

for $D=3$. This agrees of course with the known results $[4,5]$.

We can also add a Maxwell term, $-\frac{1}{4} \int \sqrt{-g} F_{\mu \nu}^{2}$, to the Einstein action to obtain the RN solution. A spherically symmetric and static (Birkhoff's theorem is also immediate here) gauge field can be written as $A=A_{0}(r) d t$, which then leads to the reduced cosmological EinsteinMaxwell system:

$$
I=\int_{0}^{\infty} d r\left\{r^{D-3}\left(a-1-\frac{\Lambda r^{2}}{D-2}\right) b^{\prime}-\frac{1}{2 b} r^{D-2} A_{0}^{\prime 2}\right\}
$$

Varying the action with respect to $A_{0}$, one obtains

$$
A_{0}=\frac{b(r) c_{3}}{r^{D-3}}
$$

The remaining equations follow easily and lead to RNdS:

$$
b(r)=c_{2}, \quad a(r)=1+\frac{c_{1}}{r^{D-3}}+\frac{\Lambda}{D-2} r^{2}+\frac{q^{2}}{r^{2 D-6}}
$$


Next, we run through some other gravitational models of current interest.

\section{Birkhoff Theorem in Einstein-Gauss-Bonnet (EGB) theory}

The EGB model frequently arises in the gravity and string theory literature. Its spherically symmetric solutions were given in [6] using in fact the Weyl trick, but assuming $t$-independence from the outset. Here, we derive the latter. The action

$$
I=\int d^{D} x \sqrt{-g}\left\{R+\alpha\left(R^{2}-4 R_{\mu \nu}^{2}+R_{\mu \nu \sigma \rho}^{2}\right)\right\}
$$

reduces to the following simple form for the metric (3)

$$
I=\int_{0}^{\infty} d r r^{D-3}(a-1) b^{\prime}\left\{1+\tilde{\alpha} r^{-2}(a-1)\right\}
$$

where $\tilde{\alpha}$ vanishes for $D=4$ and otherwise is proportional to $\alpha$. As in the case of pure Einstein theory, the action indeed has no time derivatives. The explicit solution of [6]

$$
a(r)=1+\frac{r^{2}}{2 \tilde{\alpha}} \pm \frac{r^{2}}{2 \tilde{\alpha}} \sqrt{1+\frac{c_{1} \tilde{\alpha}}{r^{D-1}}}
$$

then follows directly. To complete our discussion of EGB theory, let us add the Maxwell term,

$$
I=\int_{0}^{\infty} d r\left\{r^{D-3}(a-1) b^{\prime}\left[1+\tilde{\alpha} r^{-2}(a-1)\right]-\frac{1}{2 b} r^{D-2} A_{0}^{\prime 2}\right\}
$$

Then the "RNEGB" solution reads :

$$
b(r)=c, \quad a(r)=1+\frac{r^{2}}{2 \tilde{\alpha}} \pm \frac{r^{2}}{2 \tilde{\alpha}} \sqrt{1+\frac{c_{1} \tilde{\alpha}}{r^{D-1}}+\frac{q^{2} \tilde{\alpha}}{r^{2 D-8}}}
$$

\section{II $C^{2}$ and $C^{3}$ models}

Explicit spherically symmetric solutions of conformal, $C^{2}$, gravity were given in $[7,8]$. Birkhoff's theorem was also demonstrated in [7]. Here, we show how the Weyl trick provides us with a shortcut to the explicit solution. Dropping time dependence for simplicity, the static metric given in (1) reduces the action

$$
I=\int d^{4} x \sqrt{-g} C_{\mu \nu \rho \sigma} C^{\mu \nu \rho \sigma}
$$


to the 1-dimensional form

$$
I=\int_{0}^{\infty} d r \frac{1}{b r^{2}}\left\{r^{2} b a^{\prime \prime}+3 r^{2} a^{\prime} b^{\prime}+2 r^{2} a b^{\prime \prime}-2 r b a^{\prime}-2 a r b^{\prime}-2 b+2 b a\right\}^{2}
$$

After varying (18) with respect to $(a, b)$, one can set $b=c$ and integrate one of the two equations to obtain

$$
a(r)=c_{1}+\frac{c_{2}}{r}+c_{3} r+c_{4} r^{2}
$$

The remaining equation then imposes the following condition on the integration constants,

$$
1-c_{1}^{2}+3 c_{2} c_{3}=0
$$

The above solution seemingly accommodates both the Schwarzschild and the linear term $c_{3} r$ : However, if both were present simultaneously, the condition (20) would imply that $c_{1} \neq 1$, namely a conical singularity (and not merely a horizon). Thus, one must set $c_{1}=1$ to avoid the singularity and impose the stronger version of the condition (20), thereby excluding either the linear potential term or $1 / r$.

Let us next sketch the spherically symmetric solutions of the cubic conformal curvature model,

$$
I=\int d^{4} x \sqrt{-g} C_{\mu \nu \rho \sigma} C_{\alpha \beta}^{\mu \nu} C^{\alpha \beta \rho \sigma}
$$

which, for the metric (1), reduces to

$$
I=\int_{0}^{\infty} d r \frac{1}{b^{2} r^{4}}\left\{r^{2} b a^{\prime \prime}+3 r^{2} a^{\prime} b^{\prime}+2 r^{2} a b^{\prime \prime}-2 r b a^{\prime}-2 a r b^{\prime}-2 b+2 b a\right\}^{3} .
$$

Once again, after varying the action with respect to $(a, b)$ and setting ${ }^{6} b=c$, one can show that there can be no $M / r$ term in the metric: The Schwarzschild metric is not a solution. Instead, one can see that $a(r)=1+c_{1} r+c_{2} r^{2}$, solves the equations: It yields, as expected, the most general conformally flat metric (in the spherical coordinates). To our knowledge, this is the simplest

\footnotetext{
${ }^{6}$ In Einstein theory, $b=c$ is a consequence of the field equations. Here and below, we select this class without checking whether it is still forced. In either case, the metrics so obtained are still solutions of course.
} 
gravitational model in $D=4$ that actually excludes Schwarzschild horizons, an exclusion that also extends to the generic $R+R^{2}+C^{3}$ cases as well, since the other terms vanish for Schwarzschild metrics.

\section{Solutions of pure $R^{2}$ theory}

Let us consider the action

$$
\begin{aligned}
& I=\int d^{D} x \sqrt{-g} R^{2} \\
& \rightarrow \int d r r^{D-2} b\left\{a^{\prime \prime}+\frac{2 a b^{\prime \prime}}{b}+\frac{3 a^{\prime} b^{\prime}}{b}+2(D-2) \frac{1}{r}\left(a^{\prime}+\frac{a b^{\prime}}{b}\right)+(D-2)(D-3) \frac{1}{r^{2}}(a-1)\right\}^{2}
\end{aligned}
$$

$D=4$ is special, since the action is scale invariant and allows the following solution

$$
a(r)=1+\frac{2 M}{r}+\frac{q^{2}}{r^{2}}+\Lambda r^{2}, \quad b(r)=c,
$$

with the interesting constraint

$$
q^{2} \Lambda=0
$$

Therefore, as in $C^{2}$ theory, there is a nice complementarity here: Either a charge or a cosmological term can be present, but not both.

For generic $D$, the equations derived from (24) allow only the RN solution

$$
a(r)=1+\frac{2 M}{r}+\frac{q^{2}}{r^{2 D-6}}
$$

since scale invariance is lost in $D \neq 4$.

Let us go back to $D=4$ and study another 'special' quadratic model in $D=4$.

$$
I=\int d^{4} x \sqrt{-g} \tilde{R}_{\mu \nu} \tilde{R}^{\mu \nu}
$$

where $\tilde{R}^{\mu \nu}=R_{\mu \nu}-\frac{1}{4} g_{\mu \nu} R$. What makes this model unique among the quadratic ones is that it is the only one whose solutions have vanishing energy [9]. [ From the Gauss-Bonnet identity, this model (27) is equivalent to $\int d^{4} x \sqrt{-g} R_{\mu \nu \alpha \beta} R^{\mu \nu \alpha \beta}$ theory. Beyond $D=4$, the 'traceless' theory 
$\int d^{4} x \sqrt{-g}\left\{R_{\mu \nu}-\frac{1}{D} g_{\mu \nu} R\right\}^{2}$ keeps its 'zero-energy' property, but $\int d^{4} x \sqrt{-g} R_{\mu \nu \alpha \beta}^{2}$ does not.] The reduced action, not necessarily in the simplest form, is

$$
\begin{aligned}
I=\int_{0}^{\infty} d r \quad\{ & \frac{4 b}{r^{2}}(a-1)^{2}+\frac{r^{2}}{b}\left(2 a b^{\prime \prime}+3 a^{\prime} b^{\prime}\right)^{2}+4 r^{2} a a^{\prime \prime} b^{\prime \prime} \\
& \left.+6 r^{2} a^{\prime} b^{\prime} a^{\prime \prime}+r^{2} b a^{\prime \prime 2}+8 \frac{a^{2} b^{\prime 2}}{b}+8 a a^{\prime} b^{\prime}+4 b a^{\prime 2}\right\} .
\end{aligned}
$$

After varying with respect to $(a, b)$, and setting $b=c$, one obtains two nonlinear equations satisfied by $a$, one of which is easily integrable with 4 integration constants. But the other equation requires 2 of those constants to be zero and one is left with only SdS. While the fact that SdS is a solution of the model (27) is also easy to see without using the Weyl trick, the latter allowed us to show that among the solutions of the type $-g_{00}=g^{r r}$, SdS is the only one! One could of course remove this restriction and try to solve the two highly non-linear equations in $(a, b)$. We do not know any exact solutions of this kind ${ }^{7}$ but the reduced action (29) is a good starting point in looking for such geometries.

\section{$3 \quad$ Axial Symmetry}

As the history of solutions in GR shows, things rapidly become more complicated with decreasing Killing directions : In $D=4$, the evolution from Schwarzschild to Kerr took some 45 years. For now, we just consider the simpler $D=3$ case, to obtain its (well-known) Kerr [4] or AdS Kerr [5] solutions. Here we take the time-dependent axially symmetric metric to be

$$
d s^{2}=-a(r, t) d t^{2}+b(r, t) d r^{2}+2 k(r, t) d t d \phi+h(r, t) d \phi^{2} .
$$

Birkhoff's theorem again easily emerges in this "spin 1" world -there are no explicit time derivatives in the actions, even though the Ricci tensor and scalar have many time dependent terms. As a result, the action, with a cosmological constant, reduces to

$$
I=\int_{0}^{\infty} d r\left\{\frac{a^{\prime} h^{\prime}+k^{\prime 2}}{\sqrt{a b h+b k^{2}}}+\Lambda \sqrt{a b h+b k^{2}}\right\}
$$

\footnotetext{
${ }^{7}$ Approximate solutions were found (not via Weyl trick) for $R+\alpha R_{\mu \nu \alpha \beta}^{2}$ in [10]
} 
up to boundary terms

$$
\Delta I=\int d r B^{\prime} \quad B=-\frac{\left(f h+k^{2}\right)^{\prime}}{\sqrt{a b h+b k^{2}}}
$$

We again fix the Schwarzschild gauge by setting $h(r)=r^{2}$. Then, varying the three remaining functions yields the solution

$$
k=-J, \quad a(r)=-c-\Lambda r^{2} \quad b^{-1}(r)=-c-\Lambda r^{2}+\frac{J^{2}}{4 r^{2}} .
$$

The $\Lambda=0$ limit is the extension ( in slightly different coordinates ) of the original massless spinning metric of [4], to include mass; if we keep $\Lambda$, we get the BTZ metric [5].

\section{Summary}

We have attempted to highlight the Weyl method, within its legitimate scope, as a useful tool both for probing general properties such as Birkhoff's theorem and obtaining explicit solutions in the highly symmetric cases where they are plausibly available in general gravity models. Our explicit examples were primarily spherically, but also axially ,symmetric worlds ; the latter were also easily shown to exhibit time-independence. Other, as yet unexplored, possible applications include brane-metrics, and non-abelian gauge theories.

We thank R. S. Palais for patient discussions of his work and N. Wyllard for algebraic assistance. This research was supported by NSF Grant 99-73935

\section{References}

[1] H. Weyl, "Space-time-matter", New York: Dover 1951.

[2] R. S. Palais, "The Principle of Symmetric Criticality," Comm. Math. Physics 69, 19 (1979).

[3] M. E. Fels and C. G. Torre, "The principle of symmetric criticality in general relativity," Class. Quant. Grav. 19, 641 (2002). [arXiv:gr-qc/0108033].

[4] S. Deser, R. Jackiw and G. 't Hooft, "Three-Dimensional Einstein Gravity: Dynamics Of Flat Space," Annals Phys. 152, 220 (1984). 
[5] M. Banados, C. Teitelboim and J. Zanelli, "The Black Hole In Three-Dimensional SpaceTime," Phys. Rev. Lett. 69, 1849 (1992) [arXiv:hep-th/9204099].

[6] D. G. Boulware and S. Deser, "String Generated Gravity Models," Phys. Rev. Lett. 55, 2656 (1985).

[7] R. J. Riegert, "Birkhoff's Theorem in Conformal Gravity", Phys. Rev. Lett. 53, 315-318 (1984).

[8] P. D. Mannheim and D. Kazanas, "Exact Vacuum Solution To Conformal Weyl Gravity And Galactic Rotation Curves," Astrophys. J. 342, 635 (1989).

[9] S. Deser and B. Tekin, "Gravitational energy in quadratic curvature gravities," Phys. Rev. Lett. 89, 101101 (2002) [arXiv:hep-th/0205318]; "Energy in generic higher curvature gravity theories," Phys. Rev. D 67, 084009 (2003) [arXiv:hep-th/0212292].

[10] C. G. Callan, R. C. Myers and M. J. Perry, "Black Holes In String Theory," Nucl. Phys. B 311, 673 (1989). 